\title{
Stability Switches and Hopf Bifurcations in a Second-Order Complex Delay Equation
}

\author{
M. Roales and F. Rodríguez \\ Department of Applied Mathematics, University of Alicante, Apdo. 99, 03080 Alicante, Spain \\ Correspondence should be addressed to F. Rodríguez; f.rodriguez@ua.es
}

Received 21 July 2017; Accepted 19 September 2017; Published 15 October 2017

Academic Editor: Libor Pekař

Copyright ( 2017 M. Roales and F. Rodríguez. This is an open access article distributed under the Creative Commons Attribution License, which permits unrestricted use, distribution, and reproduction in any medium, provided the original work is properly cited.

The existence of stability switches and Hopf bifurcations for the second-order delay differential equation $x^{\prime \prime}(t)+a x^{\prime}(t-\tau)+b x(t)=$ $0, t>0$, with complex coefficients, is studied in this paper.

\section{Introduction}

The delayed friction equation

$$
x^{\prime \prime}(t)+a x^{\prime}(t)+b x^{\prime}(t-\tau)+c x(t)=0,
$$

where $c>0, \tau>0$, and $a$ and $b$ are nonnegative such that $a+b>0$, was considered by Minorsky $[1,2]$ for problems of ship stability and modeling of small vibrations of a pendulum. In $[3,4]$, the stability of the zero solution of more general forms of the delayed friction equation with real coefficients was characterized.

Delay differential equations (DDE) with complex coefficients have attracted increasing attention in the last years (e.g., [5-7]). In [8], Wei and Zhang characterized the stability of the zero solution of the retarded equation with complex coefficients

$$
x^{\prime}(t)=p x(t)+q x(t-\tau),
$$

by studying the distribution of the roots of the characteristic equation for the associated real differential system with delay and analyzed the existence of stability switches [3, 4, 9].

In [10], Li et al. presented a method for directly analyzing the stability of complex DDEs on the basis of stability switches. Their results generalize those for real DDEs, thus greatly reducing the complexity of the analysis. In [11], Roales and Rodríguez studied the stability switches of the zero solution of the neutral equation with complex coefficients

$$
x^{\prime}(t)+a x^{\prime}(t-\tau)=b x(t)+c x(t-\tau),
$$

using the results developed in [10].
The aim of this paper is to characterize the stability of the zero solution of the equation

$$
x^{\prime \prime}(t)+a x^{\prime}(t-\tau)+b x(t)=0,
$$

where $\tau>0$ is a constant delay and $a, b$ are complex parameters, with $b \neq 0$.

Using the results given by [10], the existence of stability switches and Hopf bifurcations for certain conditions on the parameters of (4) will be shown, discussing the conditions that may allow for delay dependent stabilization of the system.

\section{Methods}

To carry out our analysis, we will use some previous results that are recalled in this section (see, $[4,10,12,13]$ ).

Following [10], and similar to the analysis carried out [11] for a first-order equation, we write the characteristic equation of a time-delay system with a single delay $\tau \geq 0$ in the form

$$
\Delta(\lambda, \tau)=P(\lambda)+Q(\lambda) e^{-\lambda \tau}
$$

where $P(\lambda)$ and $Q(\lambda)$ are complex polynomial. To be able to apply the main result in [10], we will require the order of $P(\lambda)$ to be either higher than that of $Q(\lambda)$ or, if they have the same order, that $|\alpha|>|\beta|$, with $\alpha, \beta \in \mathbb{C}$ being, respectively, the highest order coefficients of $P(\lambda)$ and $Q(\lambda)$. Also, it is 
necessary that $P(\lambda)$ and $Q(\lambda)$ have no roots on the imaginary axis simultaneously and that $\lambda=0$ is not a root of (5): that is,

$$
P(0)+Q(0) \neq 0 \text {. }
$$

In the next section, it will be shown that all these conditions hold in our problem.

As shown in [10], introducing the function

$$
F(\omega)=|P(i \omega)|^{2}-|Q(i \omega)|^{2}
$$

if $\omega^{*} \neq 0$ is a zero of $F(\omega)$, then there are an infinite number of delays $\tau_{j}$ corresponding to $\omega^{*}$ satisfying

$$
\Delta\left(i \omega^{*}, \tau_{j}\right)=0
$$

Based on a previous work of Lee and Hsu [14], Li et al. established the following theorem [10, Theorem 1], characterizing, for the critical values $\tau_{j}$ such that $\Delta\left(i \omega^{*}, \tau_{j}\right)=$ 0 , the variation of the number of zeros with nonnegative real parts of $\Delta(\lambda, \tau)$, in terms of the order and sign of the first nonzero derivate of $F\left(\omega^{*}\right)$.

Theorem 1. Assume that $\Delta\left(i \omega^{*}, \tau_{j}\right)=0, j=0,1,2, \ldots$ Let $N(\tau)$ be the number of zeros with nonnegative real parts of $\Delta(\lambda, \tau)$, and let $M$ be an integer such that $F^{(M)}\left(\omega^{*}\right) \neq 0$ and $F^{m}\left(\omega^{*}\right)=0$ for all $m<M$. Then

(a) $N(\tau)$ keeps unchanged as $\tau$ increases along $\tau_{j}$ if $M$ is even,

(b) when $M$ is odd, $N(\tau)$ increases by one if $\omega^{*} F^{(M)}\left(\omega^{*}\right)>$ 0 , and decreases by one if $\omega^{*} F^{(M)}\left(\omega^{*}\right)<0$, as $\tau$ increases along $\tau_{j}$.

This theorem facilitates the stability analysis with respect to the method used in [14] and extends to the complex coefficients setting a previous result which was only valid for real DDEs [15].

Hopf bifurcation theorem gives the conditions for the existence of local nontrivial periodic solutions (e.g., [4, 12, 13]). Basic conditions are the existence of a nonzero purely imaginary root of the characteristic equation, $\lambda_{0}$, that all other eigenvalues are not integer multiples of $\lambda_{0}$, and, in addition, it must hold that, if $\alpha$ is the bifurcation parameter, the branch of eigenvalues $\lambda(\alpha)$ which satisfies $\lambda(0)=\lambda_{0}$ is such that $\operatorname{Re}\left(\lambda^{\prime}(0)\right) \neq 0$, which is called the transversality condition.

\section{Stability Analysis of the Second-Order Complex DDE}

Consider the complex DDE (4), where

$$
\begin{aligned}
& a=a_{1}+i a_{2}, \\
& b=b_{1}+i b_{2} .
\end{aligned}
$$

The characteristic equation associated with (4) is

$$
\lambda^{2}+a \lambda e^{-\lambda \tau}+b=0
$$

so that for the function $\Delta(\lambda, \tau)$, as defined in (5), one has

$$
\begin{aligned}
& P(\lambda)=\lambda^{2}+b, \\
& Q(\lambda)=a \lambda .
\end{aligned}
$$

Since $P(\lambda)$ is of higher order than $Q(\lambda)$, and since we assume $b \neq 0$, it also holds that $P(0)+Q(0) \neq 0$. Thus, the conditions to apply Theorem 1 are satisfied.

The following lemma gives $N(0)$, the number of zeros with nonnegative real parts of $\Delta(\lambda, \tau)$ when the delay is zero.

Lemma 2. Consider the complex number

$$
z=(A, B)=\left(a_{1}^{2}-a_{2}^{2}-4 b_{1}, 2 a_{1} a_{2}-4 b_{2}\right) .
$$

If $z \neq 0$ and

$$
\left|a_{1}\right|<\frac{|B|}{2 \sqrt{(-A+|z|) / 2}}
$$

then $N(0)=1$. Else, if $a_{1} \leq 0$ then $N(0)=2$, and if $a_{1}>0$ then $N(0)=0$ when $z=0$ or

$$
\left|a_{1}\right|>\frac{|B|}{2 \sqrt{(-A+|z|) / 2}},
$$

and $N(0)=1$ when $z \neq 0$ and

$$
\left|a_{1}\right|=\frac{|B|}{2 \sqrt{(-A+|z|) / 2}} .
$$

Proof. Consider the equation

$$
\Delta(\lambda, 0)=P(\lambda)+Q(\lambda)=\lambda^{2}+a \lambda+b=0 .
$$

Then,

$$
\begin{aligned}
\lambda & =\frac{-a \pm \sqrt{a^{2}-4 b}}{2} \\
& =\frac{-\left(a_{1}+i a_{2}\right) \pm \sqrt{a_{1}^{2}-a_{2}^{2}+2 i a_{1} a_{2}-4 b_{1}-4 i b_{2}}}{2} \\
& =\frac{-\left(a_{1}+i a_{2}\right) \pm \sqrt{z}}{2} .
\end{aligned}
$$

If $z=0$, there is a double root with real part $-a_{1} / 2$. If $z \neq 0$, $\lambda$ can be written as

$\lambda$

$$
=\frac{-\left(a_{1}+i a_{2}\right) \pm(B /(2 \sqrt{(-A+|z|) / 2})+i \sqrt{(-A+|z|) / 2})}{2},
$$

and the conclusion of the lemma follows.

Now consider the function $F(\omega)$ defined in (7),

$$
\begin{aligned}
F(\omega) & =\left(-\omega^{2}+b_{1}\right)^{2}+b_{2}^{2}-|a|^{2} \omega^{2} \\
& =\omega^{4}-\left(|a|^{2}+2 b_{1}\right) \omega^{2}+|b|^{2},
\end{aligned}
$$


and calculate its zeros. One gets

$$
\omega_{ \pm}^{2}=\frac{|a|^{2}+2 b_{1} \pm \sqrt{\left(|a|^{2}+2 b_{1}\right)^{2}-4|b|^{2}}}{2} .
$$

We will consider two different cases and several subcases.

Case $1\left(|a|^{2}+2 b_{1}>0\right)$

$$
\begin{aligned}
& \text { Case 1(a): }\left(|a|^{2}+2 b_{1}\right)^{2}>4|b|^{2} . \\
& \text { Case 1(b): }\left(|a|^{2}+2 b_{1}\right)^{2}=4|b|^{2} . \\
& \text { Case 1(c): }\left(|a|^{2}+2 b_{1}\right)^{2}<4|b|^{2} .
\end{aligned}
$$

Case $2\left(|a|^{2}+2 b_{1} \leq 0\right)$. First, we assume that $|a|^{2}+2 b_{1}>0$ (Case 1).

If $\left(|a|^{2}+2 b_{1}\right)^{2}>4|b|^{2}($ Case $1(a))$, then $F(\omega)$ has four real roots, $\omega_{1}^{+}, \omega_{2}^{+}, \omega_{1}^{-}, \omega_{2}^{-}$, such that

$$
\begin{aligned}
& \omega_{1}^{+}>\omega_{2}^{+}>0>\omega_{2}^{-}>\omega_{1}^{-}, \\
& \omega_{1}^{+}=-\omega_{1}^{-}, \\
& \omega_{2}^{+}=-\omega_{2}^{-} .
\end{aligned}
$$

If $\left(|a|^{2}+2 b_{1}\right)^{2}=4|b|^{2}$ (Case 1(b)), then $F(\omega)$ has two double real roots, $\omega_{1}^{+}$, $\omega_{1}^{-}$, such that

$$
\begin{aligned}
& \omega_{1}^{+}>0>\omega_{1}^{-}, \\
& \omega_{1}^{+}=-\omega_{1}^{-} .
\end{aligned}
$$

If $\left(|a|^{2}+2 b_{1}\right)^{2}<4|b|^{2}($ Case $1(c))$, then $F(\omega)$ has no real root, and therefore the stability of the zero solution of (4) does not change for any $\tau>0$.

Consider now Case 1(a), where $\omega_{1}^{+}>\omega_{2}^{+}>0>\omega_{2}^{-}>$ $\omega_{1}^{-}$. Substituting $\lambda=i \omega$ into (10), and separating the real and imaginary parts, one gets

$$
\begin{aligned}
-\omega^{2}+a_{1} \omega \sin \omega \tau-a_{2} \omega \cos \omega \tau+b_{1} & =0, \\
b_{2}+a_{1} \omega \cos \omega \tau+a_{2} \omega \sin \omega \tau & =0,
\end{aligned}
$$

obtaining the following four sets of values of $\tau$ for which there are roots.

For $\omega_{1}^{+}$and $\omega_{1}^{-}$, one gets

$$
\begin{aligned}
& \cos \omega_{1}^{+} \tau=\frac{\left(-\left(\omega_{1}^{+}\right)^{2}+b_{1}\right) a_{2}-b_{2} a_{1}}{|a|^{2} \omega_{1}^{+}}, \\
& \sin \omega_{1}^{+} \tau=\frac{\left(\left(\omega_{1}^{+}\right)^{2}-b_{1}\right) a_{1}-b_{2} a_{2}}{|a|^{2} \omega_{1}^{+}}, \\
& \cos \omega_{1}^{-} \tau=\frac{\left(-\left(\omega_{1}^{-}\right)^{2}+b_{1}\right) a_{2}-b_{2} a_{1}}{|a|^{2} \omega_{1}^{-}}, \\
& \sin \omega_{1}^{-} \tau=\frac{\left(\left(\omega_{1}^{-}\right)^{2}-b_{1}\right) a_{1}-b_{2} a_{2}}{|a|^{2} \omega_{1}^{-}} .
\end{aligned}
$$

As $\omega_{1}^{+}=-\omega_{1}^{-}$, then $\cos \omega_{1}^{+} \tau=\cos \omega_{1}^{-} \tau$ and $\sin \omega_{1}^{+} \tau=$ $-\sin \omega_{1}^{-} \tau$. By $(24) \cos \omega_{1}^{+} \tau=-\cos \omega_{1}^{-} \tau$ and $\sin \omega_{1}^{+} \tau=$ $-\sin \omega_{1}^{-} \tau$. Therefore, $\cos \omega_{1}^{+} \tau=0$ in what follows

$$
\begin{aligned}
& \tau_{n, 1}^{+}=\frac{\pi / 2}{\omega_{1}^{+}}+\frac{n \pi}{\omega_{1}^{+}} \\
& \tau_{n, 1}^{-}=\frac{-\pi / 2}{\omega_{1}^{-}}-\frac{n \pi}{\omega_{1}^{-}} . \\
& \tau_{n, 1}^{+}=\tau_{n, 1}^{-}, \quad n=0,1,2, \ldots,
\end{aligned}
$$

Similarly for $\omega_{2}^{+}$and $\omega_{2}^{-}$, we obtain the following set of values of $\tau$ for which there are roots,

$$
\begin{aligned}
\tau_{n, 2}^{+}=\tau_{n, 2}^{-}=\frac{\pi / 2}{\omega_{2}^{+}}+\frac{n \pi}{\omega_{2}^{+}}=\frac{-\pi / 2}{\omega_{2}^{-}}-\frac{n \pi}{\omega_{2}^{-}}, & \\
& n=0,1,2, \ldots .
\end{aligned}
$$

Since

$$
\begin{aligned}
F^{\prime}\left(\omega_{1}^{+}\right) & =-F^{\prime}\left(\omega_{1}^{-}\right)=2 \omega_{1}^{+}\left[2\left(\omega_{1}^{+}\right)^{2}-\left(|a|^{2}+2 b_{1}\right)\right] \\
& >0 \\
F^{\prime}\left(\omega_{2}^{+}\right) & =-F^{\prime}\left(\omega_{2}^{-}\right)=2 \omega_{2}^{+}\left[2\left(\omega_{2}^{+}\right)^{2}-\left(|a|^{2}+2 b_{1}\right)\right]
\end{aligned}
$$

$$
<0 \text {, }
$$

one has

$$
\begin{aligned}
& \omega_{1}^{+} F^{\prime}\left(\omega_{1}^{+}\right)>0 \\
& \omega_{1}^{-} F^{\prime}\left(\omega_{1}^{-}\right)>0 \\
& \omega_{2}^{+} F^{\prime}\left(\omega_{2}^{+}\right)<0 \\
& \omega_{2}^{-} F^{\prime}\left(\omega_{2}^{-}\right)<0 .
\end{aligned}
$$

Therefore, according to Theorem 1 , as $\tau$ is increased, the number of the characteristic roots with nonnegative real parts increases by two as $\tau$ passes through $\tau_{n, 1}^{+}$and decreases by two as $\tau$ passes through $\tau_{n, 2}^{+}$.

If $N(0)=0$, that is, if the zero solution of (4) is stable for $\tau=0$, as $\tau_{0,1}^{+}<\tau_{0,2}^{+}$, there are stability switches when the delays are such that

$$
\tau_{0,1}^{+}<\tau_{0,2}^{+}<\tau_{1,1}^{+}<\tau_{1,2}^{+}<\cdots
$$

Since

$$
\tau_{n+1,1}^{+}-\tau_{n, 1}^{+}=\frac{\pi / 2}{\omega_{1}^{+}}<\frac{\pi / 2}{\omega_{2}^{+}}=\tau_{n+1,2}^{+}-\tau_{n, 2}^{+},
$$

the intervals become smaller with increasing $n$, so that eventually, for a certain $k \geq 1$,

$$
\tau_{k-1,1}^{+}<\tau_{k, 1}^{+} \leq \tau_{k-1,2}^{+} .
$$

Thus, the distribution of delays is

$$
\begin{aligned}
\tau_{0,1}^{+} & <\tau_{0,2}^{+}<\tau_{1,1}^{+}<\tau_{1,2}^{+}<\cdots<\tau_{k-1,1}^{+}<\tau_{k, 1}^{+} \leq \tau_{k-1,2}^{+} \\
& <\tau_{k+1,1}^{+}<\cdots
\end{aligned}
$$


and there is only a finite number of stability switches, with the system becoming unstable for $\tau>\tau_{k-1,1}^{+}$.

If $N(0)=1$ or $N(0)=2$, the system is always unstable because $\tau_{0,1}^{+}<\tau_{0,2}^{+}$and a distribution of delays for stability switches to occur is not possible.

After the study of the stability, we wonder what happens, when there are stability switches, in the critical delays $\tau=$ $\tau_{n, j}^{ \pm}$. Denote $\lambda(\tau)=\alpha(\tau)+i \omega(\tau)$ as the root of (10) satisfying $\alpha\left(\tau_{n, j}^{ \pm}\right)=0, \omega\left(\tau_{n, j}^{ \pm}\right)=\omega_{n, j}^{ \pm}, j=1,2$. According to Theorem 1 , one has

$$
\operatorname{sgn}\left[\left(\frac{d \operatorname{Re} \lambda\left(\tau_{n, j}^{ \pm}\right)}{d \tau}\right)\right]=\operatorname{sgn}\left(\omega_{n, j}^{ \pm}\right) \operatorname{sgn} F^{\prime}\left(\omega_{n, j}^{ \pm}\right) .
$$

By (28), one gets that the transversality condition required by Hopf Theorem is satisfied. Therefore, a Hopf bifurcation occurs for these critical values.

Now we study Case $1(\mathrm{~b})$, where $\omega_{1}^{+}>0>\omega_{1}^{-}$are two real roots. Proceeding as before, there are two sets of critical values of delays $\tau_{n}^{+}$and $\tau_{n}^{-}$, corresponding to $\omega_{1}^{+}$and $\omega_{1}^{-}$, respectively, such that $\tau_{n}^{+}=\tau_{n}^{-}, n=1,2, \ldots$. Since $F^{\prime}\left(\omega_{1}^{+}\right)=F^{\prime}\left(\omega_{1}^{-}\right)=0$, we consider the second derivative,

$$
F^{\prime \prime}\left(\omega_{1}^{ \pm}\right)=4\left(|a|^{2}+2 b_{1}\right) \neq 0 .
$$

By Theorem 1, since $M=2, N(\tau)$ keeps unchanged as $\tau$ increases along $\tau_{n}$. Consequently, the stability of zero solution of (4) does not change for any $\tau>0$.

Finally, consider Case 2, where

$$
|a|^{2}+2 b_{1} \leq 0 \text {. }
$$

The function $F(\omega)$ defined in (19) has no real root, and therefore the stability of the zero solution of (4) does not change for any $\tau>0$. Thus, the following theorem has been established.

Theorem 3. Consider the second-order complex delay equation (4). The following two cases may occur concerning its stability:

(a) $\left(|a|^{2}+2 b_{1}\right)^{2}>4|b|^{2}$. In this case, if $N(0)=0$, and the distribution of delays is $0<\tau_{0,1}^{+}<\tau_{0,2}^{+}<\cdots<\tau_{k-1,1}^{+}<$ $\tau_{k, 1}^{+} \leq \tau_{k-1,2}<\tau_{k+1,1}^{+}<\cdots$, then the zero solution of (4) is asymptotically stable for $\tau \in\left(0, \tau_{0,1}^{+}\right)$and $\tau \in$ $\bigcup_{n=0}^{k-2}\left(\tau_{n, 2}^{+}, \tau_{n+1,1}^{+}\right)$, and unstable for $\tau \in \bigcup_{n=0}^{k-2}\left(\tau_{n, 1}^{+}, \tau_{n, 2}^{+}\right)$ and $\tau>\tau_{k-1,1}^{+}$. Otherwise, if $N(0)=1$ or $N(0)=2$, the zero solution of (4) is unstable for all $\tau \geq 0$.

When there are stability switches, the critical delays $\tau=$ $\tau_{n, 1}^{ \pm}, n=0 \cdots k-1$, and $\tau=\tau_{n, 2}^{ \pm}, n=0 \cdots k-2$, are Hopf bifurcation values for (4).

(b) $\left(|a|^{2}+2 b_{1}\right)^{2} \leq 4|b|^{2}$. In this case, the stability of the zero solution of (4) does not change for any $\tau>0$.

\section{Conflicts of Interest}

The authors declare that there are no conflicts of interest regarding the publication of this paper.

\section{References}

[1] N. Minorsky, "Self-excited oscillations in dynamical systems possessing retarded actions," Journal of Applied Mechanics, vol. 9, pp. A65-A71, 1942.

[2] N. Minorsky, "Experiments with activated tanks," Transactions of the American Society of Mechanical Engineers, vol. 69, pp. 735747, 1947.

[3] K. L. Cooke and Z. Grossman, "Discrete delay, distributed delay and stability switches," Journal of Mathematical Analysis and Applications, vol. 86, no. 2, pp. 592-627, 1982.

[4] Y. Kuang, Delay Differential Equations with Applications in Population Dynamics, Academic Press, New York, NY, USA, 1993.

[5] J. Y. Li and Z. H. Wang, "Local Hopf bifurcation of complex nonlinear systems with time-delay," International Journal of Bifurcation and Chaos, vol. 19, no. 3, pp. 1069-1079, 2009.

[6] Z. Zhang, C. Lin, and B. Chen, "Global stability criterion for delayed complex-valued recurrent neural networks," IEEE Transactions on Neural Networks and Learning Systems, vol. 25, no. 9, pp. 1704-1708, 2014.

[7] T. Fang and J. Sun, "Stability of complex-valued impulsive system with delay," Applied Mathematics and Computation, vol. 240, pp. 102-108, 2014.

[8] J. Wei and C. Zhang, "Stability analysis in a first-order complex differential equations with delay," Nonlinear Analysis, vol. 59, no. 5, pp. 657-671, 2004.

[9] E. Beretta and Y. Kuang, "Geometric stability switch criteria in delay differential systems with delay dependent parameters," SIAM Journal on Mathematical Analysis, vol. 33, no. 5, pp. 1144$1165,2002$.

[10] J. Li, L. Zhang, and Z. Wang, "Two effective stability criteria for linear time-delay systems with complex coefficients," Journal of Systems Science \& Complexity, vol. 24, no. 5, pp. 835-849, 2011.

[11] M. Roales and F. Rodríguez, "Stability switches in a firstorder complex neutral delay equation," Journal of Applied Mathematics, vol. 2013, 6 pages, Article ID 929186, 2013.

[12] J. K. Hale, Theory of Functional Differential Equations, Springer, New York, NY, USA, 1977.

[13] H. Smith, An Introduction to Delay Differential Equations with Applications to the Life Sciences, Springer, New York, NY, USA, 2011.

[14] M. S. Lee and C. S. Hsu, "On the $\tau$-decomposition method of stability analysis for retarded dynamical system," SIAM Journal on Control and Optimization, vol. 7, pp. 242-259, 1969.

[15] K. L. Cooke and P. van den Driessche, "On zeroes of some transcendental equations," Funkcialaj Ekvacioj, vol. 29, no. 1, pp. 77-90, 1986. 


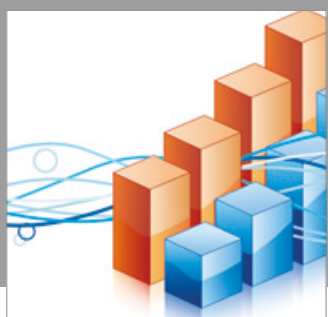

Advances in

Operations Research

vatersals

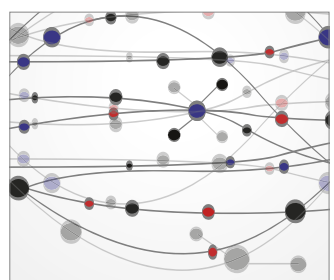

\section{The Scientific} World Journal
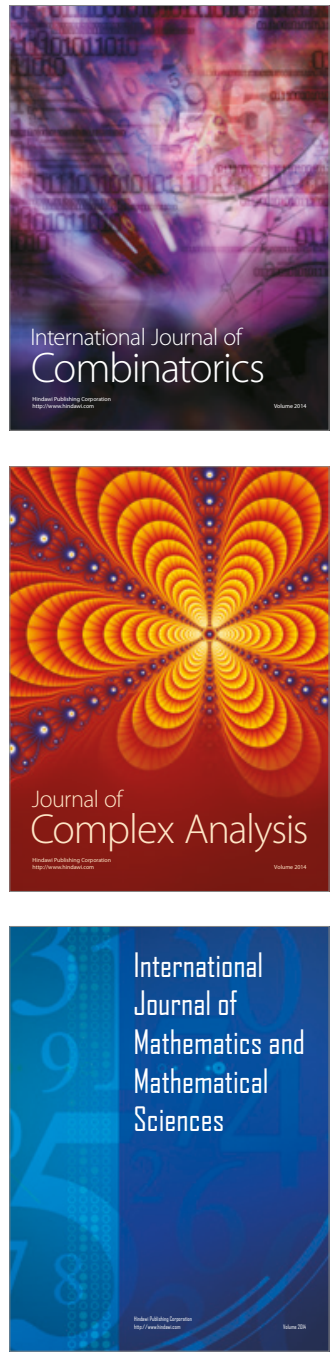
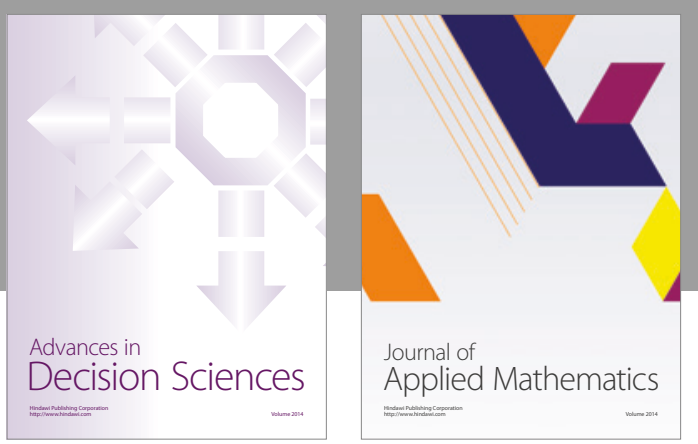

Algebra

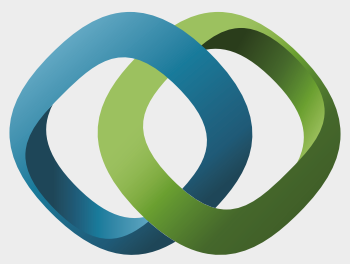

\section{Hindawi}

Submit your manuscripts at

https://www.hindawi.com
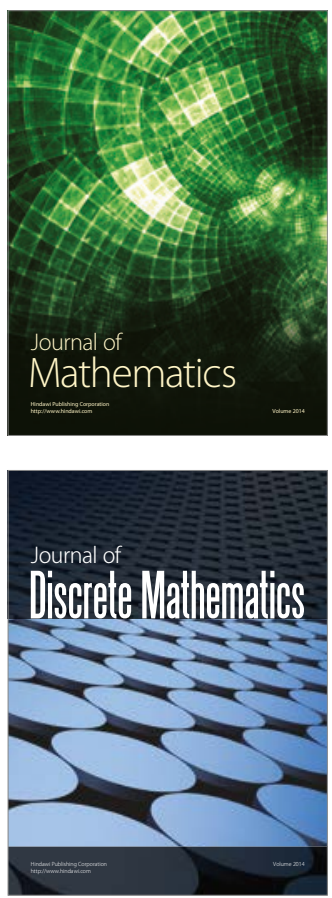

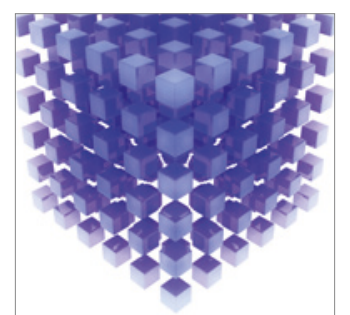

Mathematical Problems in Engineering
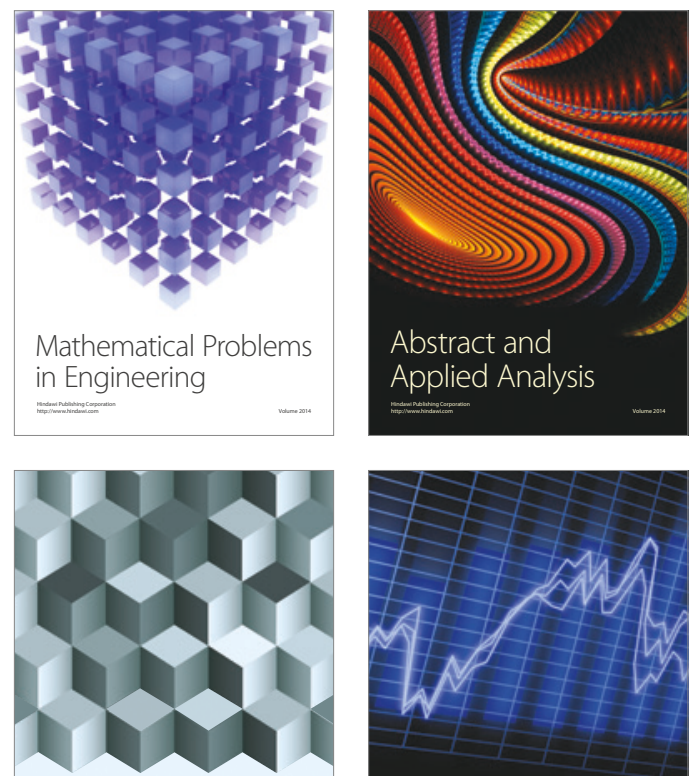

Journal of

Function Spaces

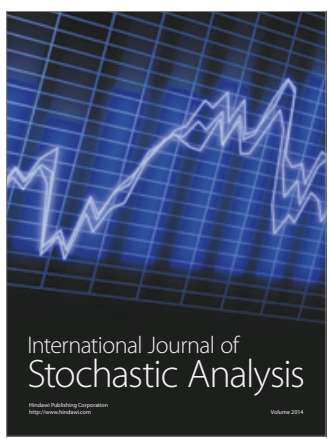

Probability and Statistics
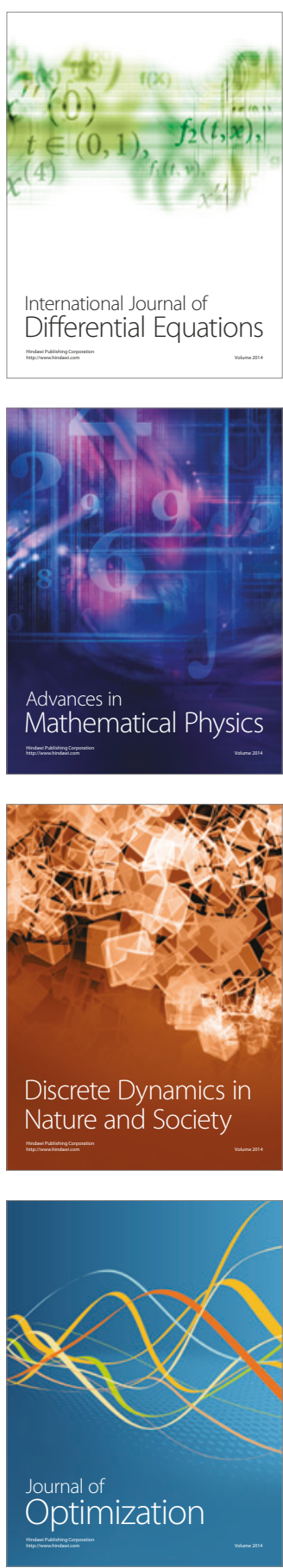